\title{
Safety and efficacy of SpyGlass cholangiopancreatoscopy in routine clinical practice in a regional Singapore hospital
}

\author{
Tiing Leong $\underline{\text { Ang }}^{1}$, FRCP, FAMS, Andrew Boon Eu $\underline{K w e k}^{1}$, MBBS, MRCP
}

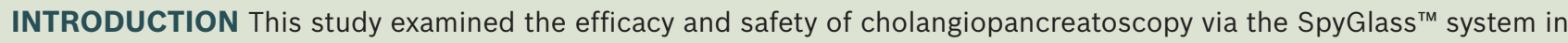
routine clinical practice.

METHODS The clinical data of endoscopic retrograde cholangiopancreatography (ERCP) performed in a regional hospital from January 2013 to November 2016 was retrieved from an electronic database and reviewed. All patients who had undergone SpyGlass cholangiopancreatoscopy were enrolled. Patient demographics, procedure indication, technical success rates, clinical success rates and complication rates were analysed. A subanalysis of clinical outcomes was performed comparing the SpyGlass legacy (fibreoptic) and digital systems.

RESULTS Out of 2,050 ERCP procedures performed, 47 patients underwent 50 cholangiopancreatoscopy procedures. Clinical indications were difficult common bile duct (CBD) stones $(59.6 \%, n=28)$, indeterminate CBD stricture $(36.2 \%$, $\mathrm{n}=17)$, indeterminate pancreatic duct stricture $(2.1 \%, \mathrm{n}=1)$ and proximally migrated CBD stent $(2.1 \%, \mathrm{n}=1)$. Complete stone extraction was achieved in 26 (92.9\%) out of 28 patients. Among patients with strictures, a correct diagnosis of malignancy based on image visualisation was achieved in all 11 cases. The sensitivity and specificity for SpyBite ${ }^{\mathrm{TM}}$ biopsies were $81.8 \%$ (95\% confidence interval [Cl] 48.2\%-97.7\%) and 100.0\% (95\% Cl 15.8\%-100.0\%), respectively. The proximally migrated CBD stent was successfully extracted. Complications included pancreatitis $(2.1 \%, n=1)$, suspected sealed perforation after laser lithotripsy treated conservatively $(2.1 \%, n=1)$ and cholangitis $(10.6 \%, n=5)$. There was no difference in clinical outcomes between the SpyGlass legacy $(n=20)$ and digital $(n=30)$ systems.

CONCLUSION SpyGlass cholangiopancreatoscopy is a safe and effective tool in routine clinical practice.

Keywords: biliary calculi, cholangiopancreatoscopy, laser lithotripsy, stricture

\section{INTRODUCTION}

Endoscopic retrograde cholangiopancreatography (ERCP) is a well-established modality for the diagnosis and management of a spectrum of benign and malignant pancreaticobiliary disorders. Common indications include common bile duct (CBD) stones and biliary obstruction from either benign or malignant disorders. ${ }^{(1)}$ In specific situations, ancillary cholangiopancreatoscopy during ERCP is required in order to directly visualise the target lesion for biopsy or definitive therapy. ${ }^{(2)}$ Cholangiopancreatoscopy during ERCP can be performed using a mother-baby scope system, an ultrathin gastroscope and the SpyGlass ${ }^{\mathrm{TM}}$ cholangioscopy system (Boston Scientific Corp, Marlboro, MA, USA). Single-operator cholangioscopy via the SpyGlass system is the simplest technique, because it uses a disposable 10-French cholangioscope inserted through the working channel of a standard duodenoscope that can be easily handled by a single endoscopist. In contrast, the mother-baby scope system requires two endoscopists to operate, while using an ultrathin gastroscope to perform ERCP is technically more difficult: as the diameter of the ultrathin gastroscope is larger, ranging from $4.9 \mathrm{~mm}$ to $5.8 \mathrm{~mm}$, it cannot be used in non-dilated ducts. ${ }^{(2)}$

Since the first report of the clinical feasibility of SpyGlass cholangioscopy in 2007, data concerning its clinical utility has been published from multiple referral centres. ${ }^{(3)}$ In the landmark initial multicentre registry study from the United States (US) and Europe with 297 patients, the overall procedure success rate was $89 \%$.
Adequate tissue was obtained for histological examination in $88 \%$ of 140 patients who underwent biopsy for stricture evaluation. Overall sensitivity was $78 \%$ for diagnosis of malignancy by visualisation but only $49 \%$ for biopsy. Procedure success, defined by protocol as visualisation and initiation of stone fragmentation and removal, was $92 \%$, although the rate of complete stone clearance during the study's SpyGlass session was lower, at $71 \% .{ }^{(4)}$ In the context of biliary stricture evaluation, Ramchandani et al ${ }^{(5)}$ showed that the accuracy of SpyGlass visualisation was $89 \%$, and in contrast to the study by Chen et al, ${ }^{(4)}$ targeted biopsies achieved a higher accuracy rate of $82 \%$. Comparing SpyGlass targeted biopsies with brush and blind biopsies, Draganov et al showed a significantly higher accuracy rate $(84.6 \%$ vs. $38.5 \%$ vs. $53.8 \%)$. ${ }^{(6)}$ Further publications confirmed the clinical utility of SpyGlass cholangioscopy in the management of a spectrum of pancreaticobiliary disorders. (7-20) $^{-20}$ Most studies have evaluated the first generation SpyGlass system, whose optical view has a somewhat limited resolution due to the analogue design of the optical probe. The new digital SpyGlass system became commercially available in 2015 and has significantly clearer optical images than the legacy (fibreoptic) SpyGlass system. Although the digital system is even easier to use due to the improved optical view and may actually achieve higher diagnostic rates in stricture evaluation, published outcome data from this system is more limited. (21)

The ease of use of SpyGlass cholangioscopy has resulted in its widespread utilisation in routine clinical practice.

${ }^{1}$ Department of Gastroenterology and Hepatology, Changi General Hospital, Singapore

Correspondence: Prof Ang Tiing Leong, Chief and Senior Consultant, Department of Gastroenterology and Hepatology, Changi General Hospital, 2 Simei Street 3, Singapore 529889. Ang.tiing.leong@singhealth.com.sg 
While excellent outcome data has been published from the pioneering referral centres, it is unclear whether similar excellent outcomes can be consistently replicated in routine clinical practice. This study examined the efficacy and safety of SpyGlass cholangiopancreatoscopy in the management of pancreaticobiliary disorders in a regional hospital in Singapore.

\section{METHODS}

This was a retrospective single-centre study conducted at Changi General Hospital, a regional hospital located in the eastern part of Singapore. This study was approved by the SingHealth Centralised Institutional Review Board.

Patients who underwent ERCP from January 2013 to November 2016 were identified from an electronic database and reviewed retrospectively. All patients who underwent SpyGlass cholangioscopy or pancreatoscopy (with or without further interventions such as targeted biopsies or laser lithotripsy) were enrolled in the study, and their clinical data was reviewed. Two groups of patients were excluded: those who underwent ERCP without the need for SpyGlass examination, and those who were initially scheduled for SpyGlass examination but did not undergo it, as the procedure was later judged to be unnecessary or technically not feasible due to anatomical abnormalities. Data such as patient demographics, procedure indication, type of SpyGlass system used, size of CBD stone, type and location of stricture, technical success, clinical success, and procedure-related complications was recorded and analysed.

ERCP with SpyGlass cholangioscopy or pancreatoscopy was performed on patients under sedation using a combination of intravenous midazolam and fentanyl administered by the endoscopist, except for one case that required anaesthetic support with intravenous propofol, as the patient could not be adequately sedated using midazolam and fentanyl. Prophylactic intravenous antibiotics were administered in all cases and continued post procedure, intravenously or orally, for at least three days. If postprocedure sepsis occurred, antibiotic treatment was extended. Selective cannulation was achieved using a papillotome and guidewire, and papillotomy was performed prior to insertion of the SpyScope ${ }^{\mathrm{TM}}$ catheter. In the context of difficult CBD stones that could not be extracted by conventional ERCP techniques such as papillotomy and mechanical lithotripsy, or balloon sphincteroplasty and extraction, stone fragmentation was achieved using Holmium-yttrium aluminium garnet laser lithotripsy. In cases of indeterminate strictures with mucosal abnormalities requiring targeted biopsies, at least six biopsies were routinely obtained using the SpyBite ${ }^{\mathrm{TM}}$ biopsy forceps.

Technical success was defined as successful insertion of the SpyGlass system to visualise the target and initiate interventions when required, such as performing targeted biopsies or initiating stone fragmentation using laser lithotripsy. A definitive diagnosis of a benign stricture was based on (a) the endoscopic appearance of a smooth surface and outline with no visible abnormal vessels; (b) benign histology if biopsies were taken and there was no progression on repeat imaging at 12-month follow-up; or (c) histology confirming its benign nature if surgery was performed. A definitive diagnosis of a malignant stricture was based on the endoscopic appearance of villous mucosal projections; irregular mucosal nodularity; mass-forming lesions; or prominent vascularisation/neo-angiogenesis and malignant histology from biopsies; surgical pathology; or, if histology was negative or unavailable, disease progression and mortality within a year of follow-up. Successful CBD stone clearance was defined as stone fragmentation and complete extraction of all stone fragments. Complications were defined using criteria previously described by Cotton et al. ${ }^{(22)}$ Serum amylase or lipase was not measured routinely after ERCP in asymptomatic patients.

The primary outcome measures were clinically successful interventions such as complete clearance of CBD stones or extraction of a migrated stent, clinical utility for the diagnosis of an indeterminate stricture, and complication rates. Secondary outcome measures were the factors associated with clinical failure and differences in clinical outcomes between the legacy and digital SpyGlass systems. The differences in clinical outcomes were analysed using chi-square or Fisher's exact test for categorical variables and Student's $t$-test for continuous variables. The sensitivity, specificity, positive predictive value and negative predictive value for the diagnosis of an indeterminate stricture were evaluated. A p-value $<0.05$ was considered as statistically significant. All statistical analysis was performed with IBM SPSS Statistics for Windows version 19.0 (IBM Corp, Armonk, NY, USA). All authors had access to study data and approved the final manuscript.

\section{RESULTS}

During the study period, a total of 2,050 ERCP procedures were performed. A total of 47 patients (mean age $63 \pm 16$ years [range 30-94 years], 55.3\% male) underwent a total of 50 SpyGlass procedures, with 44 patients undergoing one procedure and three patients undergoing two procedures. The majority (49 out of 50) of the procedures were cholangioscopy and one was pancreatoscopy. The first 20 procedures were performed using the legacy SpyGlass system and the subsequent 30 using the digital SpyGlass system when it became available. Clinical indications were: difficult CBD stones $(n=28)$, indeterminate CBD stricture $(n=17)$, indeterminate pancreatic duct stricture $(n=1)$ and proximally migrated CBD stent $(n=1)$. SpyGlass cholangiopancreatoscopy was technically successful in all cases, achieving visualisation of the target. Patient demographics and clinical data are summarised in Table I.

Among the 28 patients with CBD stones (mean size $16 \mathrm{~mm}$ [range 10-35 mm]), successful initiation of stone fragmentation by laser lithotripsy was achieved in all cases and complete stone extraction (Figs. 1-3) was achieved in 26 (92.9\%) patients. In 23 (82.1\%) patients, complete stone extraction was achieved in the index ERCP, while $3(10.7 \%)$ patients needed two sessions of cholangioscopy and laser lithotripsy prior to stone clearance. Another two patients with advanced age (87 years and 89 years) and multiple comorbidities declined repeat ERCP and were treated with long-term CBD stenting. The mean size of the CBD stone was similar in patients who had successful CBD 
Table I. Demographics and clinical data of patients who underwent SpyGlass cholangiopancreatography $(n=47)$.

\begin{tabular}{|ll|}
\hline Parameter & No. (\%) \\
\hline Age* (yr) & $63 \pm 16(30-94)$ \\
\hline Gender & \\
\hline Male & $26(55.3)$ \\
\hline Female & $21(44.7)$ \\
\hline Indication & $28(59.6)$ \\
\hline Difficult CBD stone & $17(36.2)$ \\
\hline Indeterminate CBD stricture & $1(2.1)$ \\
\hline Indeterminate pancreatic duct stricture & $1(2.1)$ \\
\hline Proximally migrated plastic CBD stent & \\
\hline Diagnosis & $28(59.6)$ \\
\hline CBD stone & $6(12.8)$ \\
\hline Benign CBD stricture & $11(23.4)$ \\
\hline Malignant/premalignant CBD stricture & $1(2.1)$ \\
\hline Benign pancreatic duct stricture & $1(2.1)$ \\
\hline Proximally migrated plastic CBD stent & \\
\hline Type of SpyGlass system & $20(40)$ \\
\hline Legacy & $30(60)$ \\
\hline Digital & \\
\hline No. of SpyGlass sessions & $44(93.6)$ \\
\hline 1 & $3(6.4)$ \\
\hline 2 & \\
\hline
\end{tabular}

*Data presented as mean \pm standard deviation (range). CBD: common bile duct

Table II. Diagnostic yield of cholangiopancreatoscopy.

\begin{tabular}{|lll|}
\hline Parameter & $\begin{array}{l}\text { True malignant } \\
\text { stricture }\end{array}$ & $\begin{array}{l}\text { True benign } \\
\text { stricture }\end{array}$ \\
\hline SpyGlass image diagnosis & & \\
\hline Benign & 0 & 7 \\
\hline Malignant & 11 & 0 \\
\hline SpyBite diagnosis & & \\
\hline Benign & 2 & 2 \\
\hline Malignant & 9 & 0 \\
\hline
\end{tabular}

clearance in one session and those who either required repeat ERCP or had failed clearance $(14.5 \mathrm{~cm}$ vs. $18.5 \mathrm{~cm} ; \mathrm{p}=0.351)$.

Among the 11 patients with biliary strictures, a correct diagnosis of malignancy based on visualisation (Figs. 4 \& 5) was achieved in all cases. All six cases of benign CBD strictures and one case of benign pancreatic duct stricture were correctly diagnosed (Table II). The sensitivity, specificity, positive predictive value and negative predictive value for SpyBite biopsies were $81.8 \%$ (95\% Cl 48.2\%-97.7\%), $100.0 \%$ (95\% Cl 15.8\%$100.0 \%), 100.0 \%(95 \% \mathrm{Cl} 66.4 \%-100.0 \%)$ and $50.0 \%(95 \%$ $\mathrm{Cl} 6.8 \%-93.2 \%$ ) respectively. One patient who had a diagnosis of malignancy based on SpyGlass cholangioscopy visualisation with inconclusive histology had disease progression and passed away five months later. Another patient with neoplastic transformation of a choledochal cyst but negative SpyBite biopsy results underwent direct peroral cholangioscopy using an ultrathin gastroscope, and histological confirmation was obtained. For the patient with proximally migrated CBD stent, SpyGlass

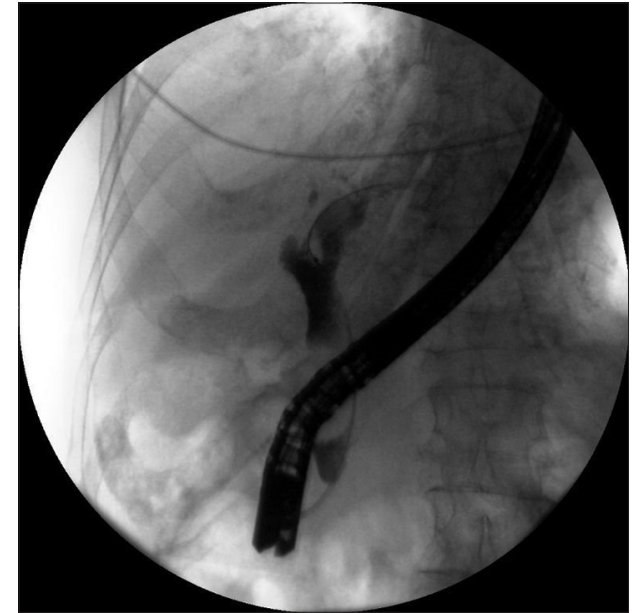

Fig. 1 Initial cholangiogram shows large bile duct stones.

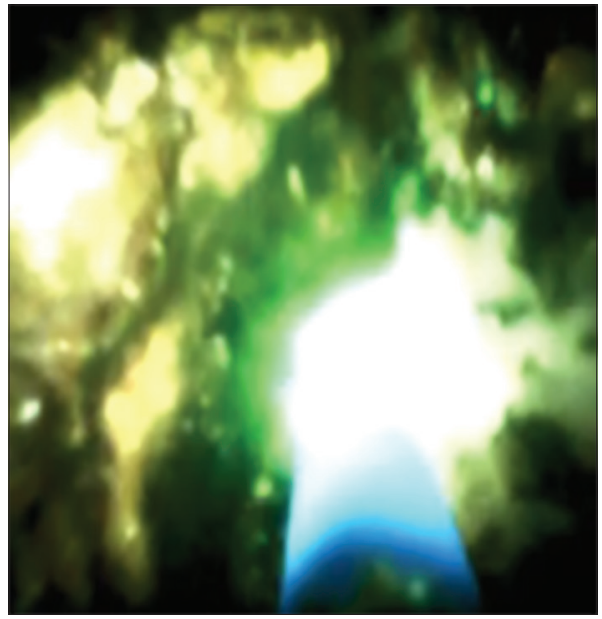

Fig. 2 Cholangioscopic image shows digital SpyGlass-directed laser lithotripsy of a bile duct stone.

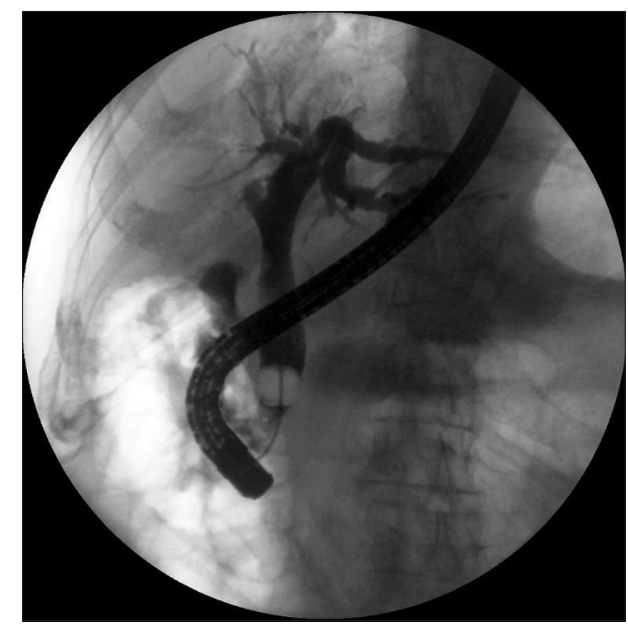

Fig. 3 Final balloon cholangiogram shows no remnant bile duct stones.

cholangioscopy facilitated insertion of a guidewire into the stent and its successful extraction with a stent retriever.

There was no difference in clinical outcome between legacy $(\mathrm{n}=20)$ and digital SpyGlass ${ }^{\mathrm{TM}}$ cholangiopancreatoscopy $(\mathrm{n}=30)$, with correct diagnosis of strictures being obtained in $5(83.3 \%)$ out of six cases and $8(88.9 \%)$ out of nine cases, respectively $(p=0.756)$, and 


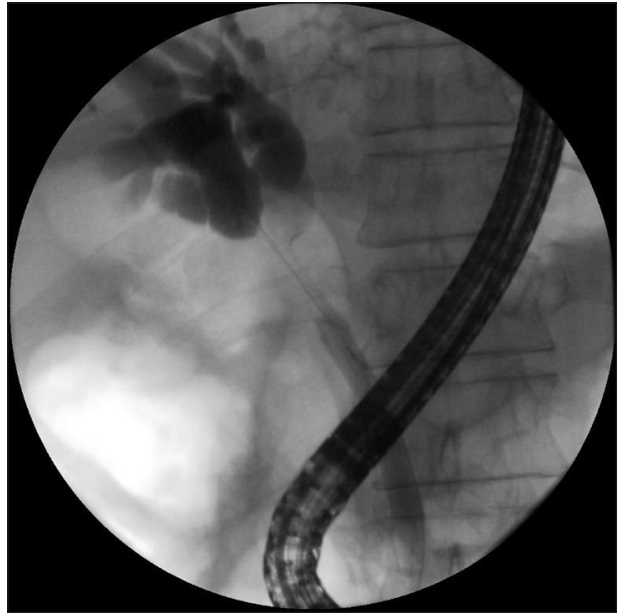

Fig. 4 Cholangiogram shows proximal bile duct stricture and dilated intrahepatic ducts.

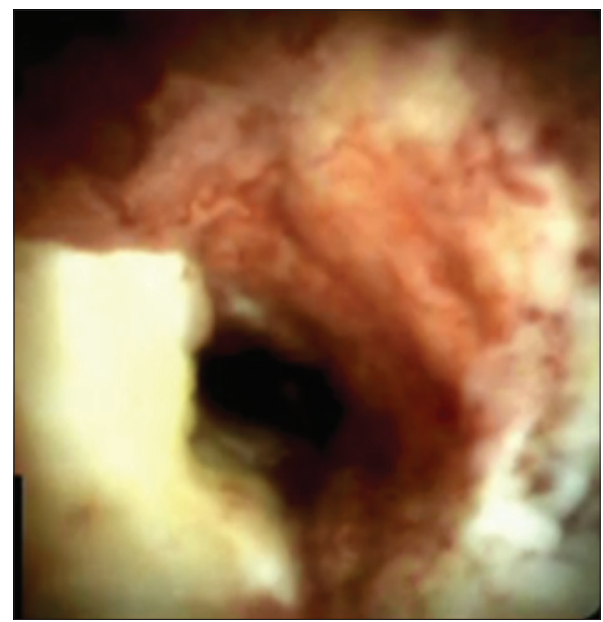

Fig. 5 Digital SpyGlass image shows cholangiocarcinoma.

complete stone extraction being achieved in 8 (88.9\%) out of nine cases and $18(94.7 \%)$ out of 19 cases, respectively $(p=0.575)$.

Postprocedure complications included $1(2.1 \%)$ case of pancreatitis, $1(2.1 \%)$ case of suspected sealed perforation after laser lithotripsy and $5(10.6 \%)$ cases of cholangitis that were successfully treated with antibiotics. The patient with suspected sealed perforation recovered and was discharged well after conservative treatment. There was no procedure-related mortality.

\section{DISCUSSION}

The introduction of the SpyGlass system revolutionised the approach towards cholangioscopy and cholangioscopic interventions. As the SpyGlass system is much easier to use compared to other cholangioscopy systems, it is more frequently utilised in routine clinical practice. Even though the system was designed as a cholangioscopy system, it can also be inserted into a sufficiently dilated pancreatic duct for interventions such as lithotripsy or biopsy. ${ }^{(2,7,10,13,23)}$ Our study was conducted in the setting of routine clinical care in a regional hospital, where SpyGlass-guided interventions were required in $2.4 \%$ of cases. Although the use of the newer digital SpyGlass system provided an improved optical view and thus made the procedure technically easier and faster, the eventual clinical outcomes of the legacy and digital systems were similar.

In the management of difficult CBD stones, complete stone clearance was achieved in $92.9 \%$ of our cases. Although some studies reported success rates of complete extraction that were somewhat lower at $71 \%-82.1 \%,{ }^{(4,7,9,16,24)}$ very high success rates of $94 \%-100 \%$ have also been reported..$^{(8,18,23,25)}$ The results of larger case series with at least ten cases are summarised in Table III. Although complete stone clearance can be achieved in a single treatment session in most cases, in the context of larger or multiple CBD stones, more than one treatment session may be required. This was consistent across both our series and other publications. . $8,18,23,25)^{2}$

In our series, the sensitivity and specificity of SpyBite biopsies for indeterminate CBD stricture were $81.8 \%$ and $100.0 \%$, respectively, while SpyGlass image-based diagnosis was correct in all cases. Hence, even though at least six biopsies were obtained in all cases in our study, there was still the problem of false negative biopsies. Nonetheless, these procedures still had higher sensitivity as compared to brush cytology and non-cholangioscopy-guided intraductal biopsies. In a recent meta-analysis, the pooled sensitivity and specificity of brush cytology for the diagnosis of malignant biliary strictures were $45 \%$ and $99 \%$, respectively, while the pooled sensitivity and specificity for non-cholangioscopy-guided intraductal biopsies were $48.1 \%$ and $99.2 \%$, respectively. A combination of both modalities only modestly increased the sensitivity to $59.4 \% .{ }^{(26)}$ In a systematic review that specifically examined the diagnostic performance of SpyGlass cholangioscopy with SpyBite biopsies, the pooled sensitivity and specificity of cholangioscopy-guided biopsies in the diagnosis of malignant biliary strictures were $60.1 \%$ and $98.0 \%$, respectively. In four studies that included patients who had previous negative imaging and brushings and/or intraductal biopsies, the pooled sensitivity and specificity for diagnosis of malignant biliary strictures were $74.7 \%$ and $93.3 \%$, respectively. ${ }^{(27)}$ SpyBite biopsies had a significantly higher sensitivity $(76.5 \%)$ than brush cytology (5.8\%) and non-cholangioscopy-guided intraductal biopsies $(29.4 \%)$ in a direct comparative study. ${ }^{(6)}$ A recent study explored the utility of rapid onsite evaluation of touch imprint cytology (ROSE-TIC) in patients undergoing SpyGlass-directed biopsies. The overall sensitivity of ROSE-TIC for diagnosing malignancy was $100.0 \%$, with a specificity of $88.9 \%$, positive predictive value of $86.7 \%$, negative predictive value of $100.0 \%$ and diagnostic accuracy of $93.5 \% .{ }^{(28)}$ This preliminary data suggested that ROSE-TIC could potentially further improve the diagnostic yield of cholangioscopy-guided biopsies.

The SpyGlass system has been successfully used for pancreatic duct interventions when the duct is sufficiently dilated to permit probe insertion. In the context of symptomatic pancreatic duct calculi, Maydeo et al successfully treated four cases using laser lithotripsy and achieved complete duct clearance; ${ }^{(23)}$ a larger US multicentre series with 28 patients reported a treatment success rate of $79 \%{ }^{(29)}$ Arnelo et al explored the use of SpyGlass pancreatoscopy in suspected intraductal papillary mucinous neoplasms (IPMN). Using pancreatoscopy, 76\% out of 17 patients 
Table III. Outcome of SpyGlass-guided electrohydraulic or laser lithotripsy in selected series of common bile duct stone disease with more than ten cases.

\begin{tabular}{|c|c|c|c|c|}
\hline Author, yr & Study design & Stone disease type, no. & SpyGlass system & Complete stone clearance \\
\hline Chen et al, $2011^{(4)}$ & Prospective multi-centre case series & Bile duct stone, 66 & Legacy & $71.2 \%$ \\
\hline $\begin{array}{l}\text { Draganov et al, } \\
2011^{(18)}\end{array}$ & Prospective single-centre case series & Bile duct stone, 26 & Legacy & $92.3 \%$ \\
\hline $\begin{array}{l}\text { Maydeo et al, } \\
2011^{(23)}\end{array}$ & Retrospective single-centre case series & $\begin{array}{l}\text { Bile duct stone, } 60 \\
\text { Pancreatic duct stone, } 4\end{array}$ & Legacy & $100.0 \%$ \\
\hline $\begin{array}{l}\text { Kalaitzakis et al, } \\
2012^{(16)}\end{array}$ & Retrospective single-centre case series & Bile duct stone, 37 & Legacy & $72.7 \%$ \\
\hline $\begin{array}{l}\text { Aljebreen et al, } \\
2014^{(8)}\end{array}$ & Retrospective single-centre case series & Bile duct stone, 13 & Legacy & $100.0 \%$ \\
\hline $\begin{array}{l}\text { Patel et al, } \\
2014^{(25)}\end{array}$ & Retrospective multi-centre case series & Bile duct stone, 69 & Legacy & $\begin{array}{l}97.1 \% \text { ( } 73.9 \% \text { extracted in } \\
1 \text { session) }\end{array}$ \\
\hline Tieu et al, $2015^{(9)}$ & Retrospective single-centre case series & Bile duct stone, 13 & Legacy & $76.9 \%$ \\
\hline $\begin{array}{l}\text { Attwell et al, } \\
2015^{(29)}\end{array}$ & Retrospective multi-centre case series & Pancreatic duct stone, 28 & Legacy & $78.5 \%$ \\
\hline $\begin{array}{l}\text { Kurihara et al, } \\
2016^{(7)}\end{array}$ & Prospective multi-centre case series & Bile duct stone, 31 & Legacy & $74.2 \%$ \\
\hline $\begin{array}{l}\text { Bhandari et al, } \\
2016^{(20)}\end{array}$ & Retrospective single-centre case series & Cystic duct stone, 34 & Legacy & $94.1 \%$ \\
\hline $\begin{array}{l}\text { Laleman et al, } \\
2017^{(24)}\end{array}$ & Retrospective single-centre case series & Bile duct stone, 39 & Legacy & $82.1 \%$ \\
\hline $\begin{array}{l}\text { Navaneethan } \\
\text { et al, } 2016^{(21)}\end{array}$ & Retrospective multi-centre case series & $\begin{array}{l}\text { Bile duct stone, } 31 \\
\text { Pancreatic duct stone, } 5\end{array}$ & Digital & $100.0 \%$ \\
\hline Current study & Retrospective single-centre case series & Bile duct stone, 28 & Legacy and digital & $92.9 \%$ \\
\hline
\end{tabular}

with a final diagnosis of main duct IPMN were correctly identified, while $78 \%$ out of nine patients with a final diagnosis of branch duct IPMN were correctly identified. Pancreatoscopy was found to have provided additional diagnostic information in the vast majority of the cases and to affect clinical decision-making in $76 \%$ of them. ${ }^{(10)}$

There is a reasonable concern that cholangioscopic or pancreatoscopic interventions may result in a higher complication rate compared to standard ERCP. Reports from studies that examined the role of cholangioscopy in indeterminate biliary strictures and difficult bile duct stones suggest that its complication rates, from complications such as pancreatitis and perforation, are similar to those of routine ERCP. In the case of pancreatic duct interventions, Arnelo et al reported that the incidence of post-ERCP pancreatitis was $17 \%$ in their cohort of patients who underwent pancreatoscopy to evaluate suspected IPMN. ${ }^{(10)}$ In the US multicentre series that evaluated laser lithotripsy for pancreatic duct stones, $8(29 \%)$ out of 28 patients had mild adverse effects, comprising 1 (4\%) patient with mild post-ERCP pancreatitis and 7 (25\%) patients with a transient increase in abdominal pain. ${ }^{(29)}$ As vigorous irrigation is required to obtain a clear view and remove debris during lithotripsy, there is the concern of increased risk of cholangitis, such that prophylactic antibiotics are routinely used during cholangioscopy. One study prospectively evaluated the risk of bacteraemia and infectious complications in 72 patients undergoing SpyGlass cholangioscopy. ${ }^{(30)}$ Blood cultures were obtained immediately before ERCP, after completion of the ERCP portion of the procedure (to determine ERCP-related bacteraemia), and 15 minutes after completion of cholangioscopy. True positive blood cultures were noted in $20(27.8 \%)$ patients, of whom $6(8.3 \%)$ patients had transient bacteraemia following ERCP. Of $14(19.4 \%)$ patients with sustained bacteraemia following ERCP or cholangioscopy, 10 (13.9\%) had sustained bacteraemia related to cholangioscopy. Despite the administration of postprocedure intravenous antibiotic, $7(9.7 \%)$ patients required further antibiotic treatment for infectious complications, 3 (4.2\%) of whom were hospitalised in order to receive intravenous antibiotic therapy. The authors concluded that the bacteraemia linked to ERCP with cholangioscopy and the subsequent risk of hospitalisation for infectious complications suggested that preprocedure antibiotic prophylaxis should be considered for patients undergoing cholangioscopy. In our study, cholangitis was the most common (10.6\%) complication despite the use of prophylactic antibiotics. A multicentre retrospective study of 282 SpyGlass cholangiopancreatoscopy procedures reported mild post-ERCP pancreatitis in $3.9 \%$, post-ERCP cholangitis in $1.4 \%$, bleeding in $1.1 \%$ and perforation in $0.7 \%$ of patients. This data was similar to that seen in large studies of ERCP performed without cholangiopancreatoscopy. ${ }^{(31)}$

The digital SpyGlass system is now available and early non-comparative data is being published. ${ }^{(21)}$ The setup is simpler and the image resolution better compared to the legacy system, making it easier and faster to visualise the target lesion. The insertion of the SpyBite forceps is also easier. The digital system further simplifies the cholangioscopy procedure and may 
potentially improve the diagnostic yield in stricture evaluation. Although treatment success rates for CBD stones are already very high with the legacy system, and hence the additional therapeutic advantage may be less, the digital system makes the process easier. In addition to the management of difficult CBD stones and indeterminate strictures, for which its utility has been established, the legacy SpyGlass system has also been successfully used in a myriad of other indications, such as to direct guidewire placement to navigate and traverse tight strictures or access the cystic duct, and to extract migrated stents; this will probably be even easier with the new digital system. ${ }^{(32-36)}$

Given the costs of the SpyGlass system, it is important to clarify how to utilise it in a cost-effective manner. It is important to decide a priori when the SpyGlass system should be used at the index ERCP, because its use can save the cost of an additional ERCP. In the case of CBD stones, the use of the SpyGlass system is clearly indicated in specific situations where the stone is too big to be captured by the mechanical lithotripsy basket or extracted by large balloon sphincteroplasty. In the context of indeterminate CBD strictures, a recent study compared the cost-effectiveness of five ERCP-based techniques for diagnosing cholangiocarcinoma in patients with primary sclerosing cholangitis-induced biliary strictures, using a Monte Carlo simulation to assess outcomes. It concluded that compared to ERCP with brush cytology; ERCP with brushing cytology and fluorescence in situ hybridisation (FISH)-trisomy; ERCP with brush cytology and FISH-polysomy; and ERCP with intraductal biopsy sampling, SpyGlass cholangioscopy with targeted biopsy was the most cost-effective diagnostic modality. ${ }^{(37)}$

We acknowledge the limitations of our study. This was a single-centre retrospective study with a limited study population. Data on procedure time and length of stay was obtained retrospectively and may not accurately reflect the time attributed to the procedure. Therefore, we did not report this data but focused on more robust outcome data such as treatment success and complication rates. In addition, most of the procedures were performed by a single endoscopist (Ang TL). Nonetheless, this data is still important, because local data has not been published and our results are consistent with the outcomes from high-volume referral centres around the world. . $8,18,21,23,25,27)$

In conclusion, our study demonstrated that SpyGlass cholangiopancreatoscopy was a safe and effective tool in routine clinical practice. It was effective in the treatment of difficult CBD stones and the evaluation of indeterminate pancreaticobiliary strictures.

\section{REFERENCES}

1. Adler DG, Lieb JG 2nd, Cohen J, et al. Quality indicators for ERCP. Gastrointest Endosc 2015; 81:54-66.

2. ASGE Technology Committee, Komanduri S, Thosani N, et al. Cholangiopancreatoscopy. Gastrointest Endosc 2016; 84:209-21.

3. Chen YK, Pleskow DK. SpyGlass single-operator peroral cholangiopancreatoscopy system for the diagnosis and therapy of bile-duct disorders: a clinical feasibility study (with video). Gastrointest Endosc 2007; 65:832-41.

4. Chen YK, Parsi MA, Binmoeller KF, et al. Single-operator cholangioscopy in patients requiring evaluation of bile duct disease or therapy of biliary stones (with videos). Gastrointest Endosc 2011; 74:805-14.
5. Ramchandani M, Reddy DN, Gupta R, et al. Role of single-operator peroral cholangioscopy in the diagnosis of indeterminate biliary lesions: a single-center, prospective study. Gastrointest Endosc 2011; 74:511-9.

6. Draganov PV, Chauhan S, Wagh MS, et al. Diagnostic accuracy of conventional and cholangioscopy-guided sampling of indeterminate biliary lesions at the time of ERCP: a prospective, long-term follow-up study. Gastrointest Endosc 2012; 75:347-53.

7. Kurihara T, Yasuda I, Isayama H, et al. Diagnostic and therapeutic single-operator cholangiopancreatoscopy in biliopancreatic diseases: prospective multicenter study in Japan. World J Gastroenterol 2016; 22:1891-901.

8. Aljebreen AM, Alharbi OR, Azzam N, Almadi MA. Efficacy of spyglass-guided electrohydraulic lithotripsy in difficult bile duct stones. Saudi J Gastroenterol 2014; 20:366-70.

9. Tieu AH, Kumbhari V, Jakhete N, et al. Diagnostic and therapeutic utility of SpyGlass $(\circledR)$ peroral cholangioscopy in intraductal biliary disease: single-center, retrospective, cohort study. Dig Endosc 2015; 27:479-85.

10. Arnelo U, Siiki A, Swahn F, et al. Single-operator pancreatoscopy is helpful in the evaluation of suspected intraductal papillary mucinous neoplasms (IPMN). Pancreatology 2014; 14:510-4.

11. Siiki A, Rinta-Kiikka I, Koivisto $\mathrm{T}$, et al. Spyglass single-operator peroral cholangioscopy seems promising in the evaluation of primary sclerosing cholangitis-related biliary strictures. Scand J Gastroenterol 2014; 49:1385-90.

12. Rey JW, Hansen T, Dümcke S, et al. Efficacy of SpyGlass (TM)-directed biopsy compared to brush cytology in obtaining adequate tissue for diagnosis in patients with biliary strictures. World J Gastrointest Endosc 2014; 6:137-43.

13. Nagayoshi Y, Aso T, Ohtsuka T, et al. Peroral pancreatoscopy using the SpyGlass system for the assessment of intraductal papillary mucinous neoplasm of the pancreas. J Hepatobiliary Pancreat Sci 2014; 21:410-7.

14. Theodoropoulou A, Vardas E, Voudoukis E, et al. SpyGlass Direct Visualization System facilitated management of iatrogenic biliary stricture: a novel approach in difficult cannulation. Endoscopy 2012; 44 Suppl 2:E433-4.

15. Manta R, Frazzoni M, Conigliaro R, et al. SpyGlass single-operator peroral cholangioscopy in the evaluation of indeterminate biliary lesions: a single-center, prospective, cohort study. Surg Endosc 2013; 27:1569-72.

16. Kalaitzakis E, Webster GJ, Oppong KW, et al. Diagnostic and therapeutic utility of single-operator peroral cholangioscopy for indeterminate biliary lesions and bile duct stones. Eur J Gastroenterol Hepatol 2012; 24:656-64.

17. Siddiqui AA, Mehendiratta V, Jackson W, et al. Identification of cholangiocarcinoma by using the Spyglass Spyscope system for peroral cholangioscopy and biopsy collection. Clin Gastroenterol Hepatol 2012; 10:466-71.

18. Draganov PV, Lin T, Chauhan S, et al. Prospective evaluation of the clinical utility of ERCP-guided cholangiopancreatoscopy with a new direct visualization system. Gastrointest Endosc 2011; 73:971-9.

19. Kantsevoy SV, Frolova EA, Thuluvath PJ. Successful removal of the proximally migrated pancreatic winged stent by using the SpyGlass visualization system. Gastrointest Endosc 2010; 72:454-5.

20. Bhandari S, Bathini R, Sharma A, Maydeo A. Usefulness of single-operator cholangioscopy-guided laser lithotripsy in patients with Mirizzi syndrome and cystic duct stones: experience at a tertiary care center. Gastrointest Endosc 2016; 84:56-61.

21. Navaneethan U, Hasan MK, Kommaraju K, et al. Digital, single-operator cholangiopancreatoscopy in the diagnosis and management of pancreatobiliary disorders: a multicenter clinical experience (with video). Gastrointest Endosc 2016; 84:649-55

22. Cotton PB, Lehman G, Vennes J, et al. Endoscopic sphincterotomy complications and their management: an attempt at consensus. Gastrointest Endosc 1991; 37:383-93.

23. Maydeo A, Kwek BE, Bhandari S, Bapat M, Dhir V. Single-operator cholangioscopy-guided laser lithotripsy in patients with difficult biliary and pancreatic ductal stones (with videos). Gastrointest Endosc 2011; 74:1308-14.

24. Laleman W, Verraes K, Van Steenbergen W, et al. Usefulness of the single-operator cholangioscopy system SpyGlass in biliary disease: a single-center prospective cohort study and aggregated review. Surg Endosc 2017; 31:2223-32.

25. Patel SN, Rosenkranz L, Hooks B, et al. Holmium-yttrium aluminum garnet laser lithotripsy in the treatment of biliary calculi using single-operator cholangioscopy: a multicenter experience (with video). Gastrointest Endosc 2014; 79:344-8.

26. Navaneethan $U$, Njei B, Lourdusamy $V$, et al. Comparative effectiveness of biliary brush cytology and intraductal biopsy for detection of malignant biliary strictures: a systematic review and meta-analysis. Gastrointest Endosc 2015; 81:168-76.

27. Navaneethan $U$, Hasan MK, Lourdusamy $V$, et al. Single-operator cholangioscopy and targeted biopsies in the diagnosis of indeterminate biliary strictures: a systematic review. Gastrointest Endosc 2015; 82:608-14.e2.

28. Varadarajulu S, Bang JY, Hasan MK, et al. Improving the diagnostic yield of single-operator cholangioscopy-guided biopsy of indeterminate biliary strictures: ROSE to the rescue? (with video). Gastrointest Endosc 2016; 84:681-7. 
29. Attwell AR, Patel S, Kahaleh M, et al. ERCP with per-oral pancreatoscopy-guided laser lithotripsy for calcific chronic pancreatitis: a multicenter U.S. experience. Gastrointest Endosc 2015; 82:311-8.

30. Thosani N, Zubarik RS, Kochar R, et al. Prospective evaluation of bacteremia rates and infectious complications among patients undergoing single-operator choledochoscopy during ERCP. Endoscopy 2016; 48:424-31.

31. Adler DG, Cox K, Milliken M, et al. A large multicenter study analysis of adverse events associated with single operator cholangiopancreatoscopy. Minerva Gastroenterol Dietol 2015; 61:179-84.

32. Wright H, Sharma S, Gurakar A, et al. Management of biliary stricture guided by the Spyglass Direct Visualization System in a liver transplant recipient: an innovative approach. Gastrointest Endosc 2008; 67:1201-3.

33. Woo YS, Lee JK, Noh DH, et al. SpyGlass cholangioscopy-assisted guidewire placement for post-LDLT biliary strictures: a case series. Surg Endosc 2016
30:3897-903.

34. Barkay O, Bucksot L, Sherman S. Endoscopic transpapillary gallbladder drainage with the SpyGlass cholangiopancreatoscopy system. Gastrointest Endosc 2009; 70:1039-40.

35. Maydeo A, Kwek A, Bhandari S, Bapat M, Mathew P. SpyGlass pancreatoscopy-guided cannulation and retrieval of a deeply migrated pancreatic duct stent. Endoscopy 2011; 43 Suppl 2:E137-8.

36. Kantsevoy SV, Frolova EA, Thuluvath PJ. Successful removal of the proximally migrated pancreatic winged stent by using the SpyGlass visualization system. Gastrointest Endosc 2010; 72:454-5.

37. Njei B, McCarty TR, Varadarajulu S, Navaneethan U. Cost utility of ERCP-based modalities for the diagnosis of cholangiocarcinoma in primary sclerosing cholangitis. Gastrointest Endosc 2017; 85:773-81.e10. 